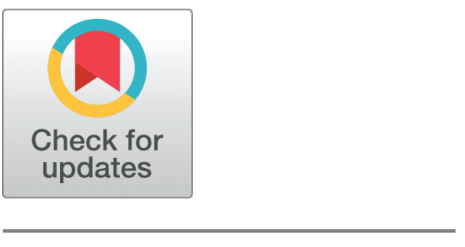

open acCess

Received: 17.07 .2020

Accepted: 26.02.2021

Published: 09.04.2021

Citation: Singla N, Kaur H (2021) A Two-State Retrial Queueing Model with Feedback having Two Identical Parallel Servers. Indian Journal of Science and Technology 14(11): 915-931. https://doi.org/ 10.17485/IJST/V14i11.1167

* Corresponding author.

Tel: +91-9465311134

harwinderkaurchahal@gmail.com

Funding: None

Competing Interests: None

Copyright: () 2021 Singla \& Kaur. This is an open access article distributed under the terms of the Creative Commons Attribution License, which permits unrestricted use, distribution, and reproduction in any medium, provided the original author and source are credited.

Published By Indian Society for Education and Environment (iSee)

ISSN

Print: 0974-6846

Electronic: 0974-5645

\section{A Two-State Retrial Queueing Model with Feedback having Two Identical Parallel Servers}

\author{
Neelam Singla ${ }^{1}$, Harwinder Kaur ${ }^{2 *}$ \\ 1 Assistant Professor, Department of Statistics, Punjabi University, Patiala, 147002, India \\ 2 Research Scholar, Department of Statistics, Punjabi University, Patiala, 147002, India. \\ Tel.: +91-9465311134
}

\section{Abstract}

Objective: In this study we consider a two-state retrial queueing system with feedback having two identical parallel servers. Transient state probabilities for exact number of arrivals and departures from the system will be obtained when both, one or none of the servers is busy. Numerical and graphical solutions will also be obtained. Methods: The difference-differential equations governing the system are solved recursively, Laplace transform is then used to obtain the transient state probabilities for exact number of arrivals and departures from the system. Findings: Time dependent probabilities are obtained when both, one and none of the servers is busy. Numerical and Graphical solutions are also obtained using MATLAB programming. Novelty: In past research, models considered arrivals and departures from the orbit whereas in present model arrivals and departures from the system are studied along with the concept of feedback. Applications: This type of model is implemented in computer systems.

Mathematics Subject Classification: 60K25, 90B22, 60M20

Keywords: Arrivals; Departures; Queueing; Retrial; Feedback

\section{Introduction}

Queueing systems with repeated calls are frequently observed in many real life situations such as telecommunication systems, computer networks and these are popularly known as retrial queueing systems. In retrial queues if a customer on arrival finds any of the servers free it is served immediately else joins the orbit (pool) and retries for service after a random amount of time. The primary and secondary arrivals both follow Poisson distribution. A basic example of retrial queueing system is the case where a person tries to connect a call but the line goes busy so the person tries to reconnect the call after some time as a retrial customer. Initial work on retrial queues was done by ${ }^{(1-4)}$. 


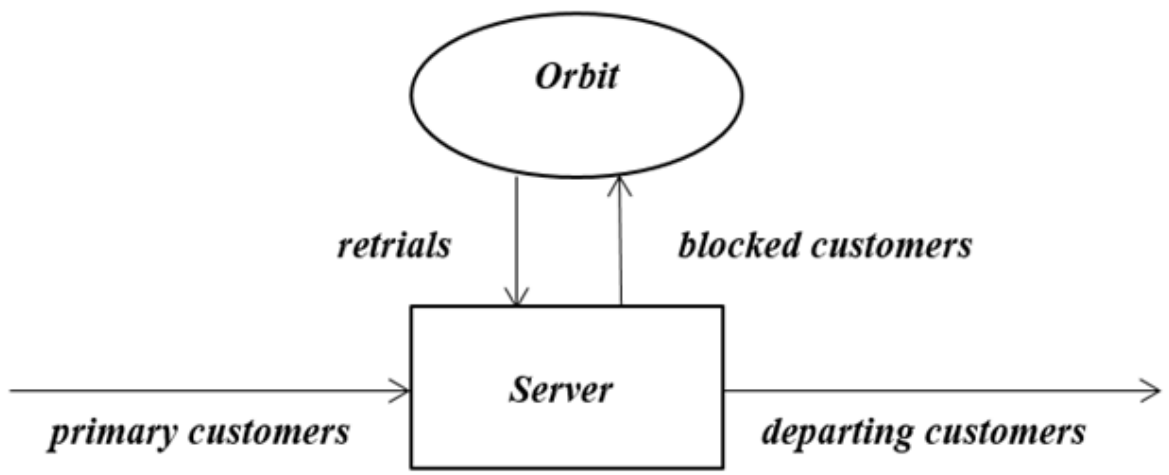

Fig 1. Basic structure of a retrial queueing system

As in retrial queueing systems it is described that a customer which do not get immediate service, will retry from the orbit after a random amount of period as a repeated call. If the customer is unsatisfied after service, he/she joins the orbit and retry for service as a feedback retrial customer. For example when a message faces failed transmission in multiple access telecommunication systems, it can be sent again. For feedback queueing systems, the pioneering work was done by ${ }^{(5)}$ in his paper 'Cyclic queues with feedback' and by ${ }^{(6)}$ in paper 'A single-server queue with feedback. ${ }^{(7)}$ discussed 'The M/G/1 feedback retrial queue with two types of customers' where the joint generating function of the number of customers in two groups are derived by using the supplementary variable method. ${ }^{(8)}$ analyzed 'A feedback retrial queuing system with starting failures and single vacation' where the system size distribution at random points and various performance measures are derived. ${ }^{(9)}$ studied 'A single server feedback retrial queue with collisions' in which the joint distribution of server state and the orbit length under steady state was studied using generating function technique. ${ }^{(10)}$ analyzed 'Finite Queueing Model with Multitask Servers and Blocking' where expressions for mean number of customers in the system, average queue length and blocking probability are obtained. ${ }^{(11)}$ worked on 'The multi-server retrial system with Bernoulli feedback and starting failures' in which formulae for calculating rate matrix and stationary probabilities were obtained by using matrix-geometric method. ${ }^{(12)}$ published 'Retrial queueing models in discrete time: a short survey of some late arrival models' where average orbit size and mean busy period are obtained from generating function ${ }^{(13)}$ analyzed 'Some new results for the M/M/1 queue' in which solution is obtained for the probability that exactly ' $i$ ' number of arrivals, ' $j$ ' number of services occur over a time interval t. ${ }^{(14)}$ worked on 'Transient analysis of markovian queueing model with bernoulli schedule and multiple working vacations' where transient state probabilities were computed. ${ }^{(15)}$ discussed 'Performance analysis of a two-state queueing model with retrials' and ${ }^{(16)}$ worked on 'A two-state multiserver queueing system with retrials' where the time-dependent probabilities were obtained.

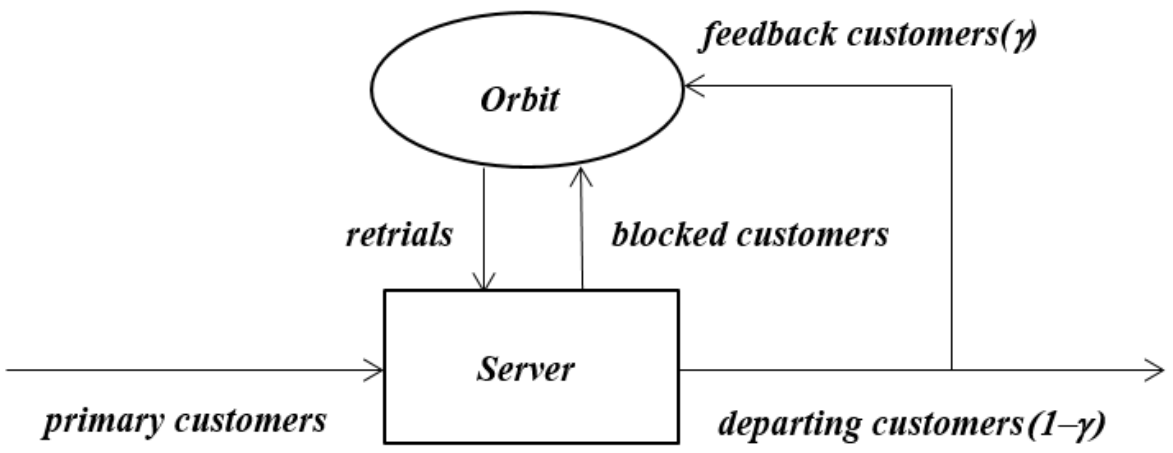

Fig 2. Basic structure of a retrial queueing system with feedback

The paper is organized in various sections as follows: The model is described mathematically in section 2 where the difference-differential equations governing the system are given. The transient state probabilities are obtained in section 3 . Various special cases are obtained in section 4 . The numerical and graphical solutions are illustrated in section 5 . The numerical and graphical representation of busy period distribution of the system and the servers is presented in section 6 . Finally, the conclusion is given in section 7 which is further followed by references. 


\section{Model Description}

The detailed description of the present model is given as follows:

- The Arrival Process: The primary customers arrive at the system according to Poisson process with mean arrival rate $\lambda$.

- The Retrial Process: If a customer on arrival finds any of the servers free, he is served immediately. Otherwise, the customer joins the orbit and calls repeatedly until he finds any of the servers free. The retrial customers arrive at the system according to Poisson process with retrial rate $\theta$.

- The Feedback Rule: If a customer is unsatisfied with the service, he may join the orbit as a feedback customer. A departing customer joins the orbit with probability $\gamma$ for receiving a satisfied service and leaves the system with probability $(1-\gamma)$.

- The Service Process: Service times follow exponential distribution with mean service rate $\mu$.

The input flow of primary calls, intervals between repetitions, service times are statistically independent.

Laplace Transformation of $\bar{f}(s)$ of $f(t)$ is given by:

$$
\bar{f}(s)=\int_{0}^{\infty} e^{-s t} f(t) d t ; \quad \operatorname{Re}(s)>0
$$

The Laplace inverse of

$$
\frac{Q(p)}{P(p)}=\sum_{k=1}^{n} \sum_{l=1}^{m_{k}} \frac{t^{m_{k}-l} e^{a_{k} t}}{\left(m_{k}-l\right) !(l-1) !} \times \frac{d^{l-1}}{d p^{l-1}}\left(\frac{Q(p)}{P(p)}\right)\left(p-a_{k}\right)^{m_{k}} \forall \mathrm{p}=a_{k}, \quad a_{i} \neq a_{k} \text { for } \mathrm{i} \neq \mathrm{k}
$$

Where, $\mathrm{P}(\mathrm{p})=\left(p-a_{1}\right)^{m_{1}}\left(p-a_{2}\right)^{m_{2}} \ldots \ldots \ldots\left(p-a_{n}\right)^{m_{n}}$

$\mathrm{Q}(\mathrm{p})$ is a polynomial of degree $<<m_{1}+m_{2}+m_{3}+\ldots \ldots \ldots \ldots m_{n}-1$.

The Laplace inverse of $\bar{N}_{n_{1, n_{2}, n_{3}}}^{a, b, c}(\mathrm{~s})=\frac{1}{(s+a)^{n_{1}}(s+b)^{n_{2}}(s+c)^{n_{3}}}$ is is

$$
\begin{aligned}
N_{n_{1, n_{2}, n_{3}}^{a}, b, c}^{a}= & \sum_{l=1}^{n_{3}} \sum_{m=1}^{l} \frac{e^{-a t} t^{n_{3}-l}(-1)^{m+1}\left(\frac{l-1}{m-1}\right)\left(\prod_{g_{1}=0}^{l-m-1}\left(n_{1}+g_{1}\right)\right)\left(\prod_{g_{2}=0}^{m-2}\left(n_{2}+g_{2}\right)\right)}{\left(n_{3}-l\right) !(m-1) !(b-a)^{n_{2}+m-1}(c-a)^{n_{1}+l-m}} \\
& +\sum_{l=1}^{n_{2}} \sum_{m=1}^{l} \frac{e^{-b t} t^{n_{2}-l}(-1)^{m+1}\left(\frac{l-1}{m-1}\right)\left(\prod_{g_{1}=0}^{l-m-1}\left(n_{1}+g_{1}\right)\right)\left(\prod_{g_{2}=0}^{m-2}\left(n_{3}+g_{2}\right)\right)}{\left(n_{2}-l\right) !(m-1) !(a-b)^{n_{3}+m-1}(c-b)^{n_{1}+l-m}} \\
& +\sum_{l=1}^{n_{1}} \sum_{m=1}^{l} \frac{e^{-c t} t^{n_{1}-l}(-1)^{m+1}\left(\frac{l-1}{m-1}\right)\left(\prod_{g_{1}=0}^{l-m-1}\left(n_{2}+g_{1}\right)\right)\left(\prod_{g_{2}=0}^{m-2}\left(n_{3}+g_{2}\right)\right)}{\left(n_{1}-l\right) !(m-1) !(a-c)^{n_{3}+m-1}(b-c)^{n_{2}+l-m}}
\end{aligned}
$$

If $L^{-1}\{\mathrm{f}(\mathrm{s})\}=\mathrm{F}(\mathrm{t})$ and $L^{-1}\{\mathrm{~g}(\mathrm{~s})\}=\mathrm{G}(\mathrm{t})$ then

$$
L^{-1}\{\mathrm{f}(\mathrm{s}) \mathrm{g}(\mathrm{s})\}=\int_{0}^{t} F(\mathrm{u}) G(\mathrm{t}-\mathrm{u}) \mathrm{du}=\mathrm{F}^{*} \mathrm{G}
$$

$F^{*} \mathrm{G}$ is called the convolution of $\mathrm{F}$ and $\mathrm{G}$.

\subsection{The Two-Dimensional State Model}

\subsubsection{Definitions}

$P_{i, j, 0}(t)=$ Probability that there are exactly i number of arrivals, $\mathrm{j}$ number of departures by time $\mathrm{t}$ and servers are free.

$P_{i, j, k}(t)=$ Probability that there are exactly i number of arrivals, $\mathrm{j}$ number of departures from the system by time $\mathrm{t}$ when $\mathrm{k}(\mathrm{k}$ $=1$ or 2 ) servers are busy.

$P_{i, j}(t)=$ Probability that there are exactly i number of arrivals, $\mathrm{j}$ number of departures from the system by time $\mathrm{t}$.

$$
P_{i, j}(t)=P_{i, j, 0}(t)+P_{i, j, 1}(t)+P_{i, j, 2}(t), \forall i, j ; i \geq j
$$

Initially

$$
P_{0,0,0}(0)=1 ; P_{i, j, 0}(t)=0, i<j ; P_{i, j, k}(t)=0, i<j P_{i, 0, k}=0, i \geq 0,(k=1 \text { or } 2)
$$




\subsubsection{The Difference Differential equations governing the system are:}

$$
\begin{gathered}
\frac{d}{d t} P_{i, j, 0}(t)=-(\lambda+(i-j) \theta) P_{i, j, 0}(t)+\mu(1-\gamma) P_{i, j-1,1}(t)+\mu \gamma P_{i, j, 1}(t) ; i \geq j \geq 0 \\
\frac{d}{d t} P_{i, j, 1}(t)=-(\lambda+\mu+(i-j-1) \theta) P_{i, j, 1}(t)+\lambda P_{i-1, j, 0}(t)+(i-j) \theta P_{i, j, 0}(t)+2 \mu(1-\gamma) P_{i, j-1,2}(t)+2 \mu \gamma P_{i, j, 2}(t) ; i>j \geq 0 \\
\frac{d}{d t} P_{i, j, 2}(t)=-(\lambda+2 \mu) P_{i, j, 2}(t)+\lambda P_{i-1, j, 1}(t)+\lambda P_{i-1, j, 2}(t)+(i-j-1) \theta P_{i, j, 1}(t) ; i>1, i>j \geq 0
\end{gathered}
$$

Using Laplace Transform $\bar{f}(s)$ of $f(t)$ given by

$$
\bar{f}(s)=\int_{0}^{\infty} e^{-s t} f(t) d t ; \quad \operatorname{Re}(s)>0
$$

and using initial condition in equations (1) to (3), we have

$$
\begin{gathered}
(s+\lambda+(i-j) \theta) \bar{P}_{i . j .0}(s)=\mu(1-\gamma) \bar{P}_{i, j-1,1}(s)+\mu \gamma \bar{P}_{i, j, 1}(s) ; i \geq j \geq 0 \\
(s+\lambda+\mu+(i-j-1) \theta) \bar{P}_{i, j, 1}(s)=\lambda \bar{P}_{i-1, j, 0}(s)+(i-j) \theta \bar{P}_{i, j, 0}(s)+2 \mu(1-\gamma) \bar{P}_{i, j-1,2}(s)+2 \mu \gamma \bar{P}_{i, j, 2}(s) ; i>j \geq 0 \\
(s+\lambda+2 \mu) \bar{P}_{i, j, 2}(s)=\lambda \bar{P}_{i-1, j, 1}(s)+\lambda \bar{P}_{i-1, j, 2}(s)+(i-j-1) \theta \bar{P}_{i, j, 1}(s) ; i>j \geq 0
\end{gathered}
$$

\section{Solution of the Problem}

Solving equations (4) to (6) recursively, we have

$$
\begin{gathered}
\bar{P}_{0,0,0}(s)=\frac{1}{s+\lambda} \\
\bar{P}_{1,0,1}(s)=\frac{\lambda}{s+\lambda+\mu} \bar{P}_{0,0,0}(s)+\frac{\theta}{s+\lambda+\mu} \bar{P}_{1,0,0}(s) \\
\bar{P}_{1,1,0}(s)=\frac{\mu(1-\gamma)}{s+\lambda} \bar{P}_{1,0,1}(s) \\
\bar{P}_{i, i, 0}(s)=\frac{\mu(1-\gamma)}{s+\lambda}\left\{\frac{\lambda}{s+\lambda+\mu} \bar{P}_{i-1, i-1,0}(s)+\frac{\theta}{s+\lambda+\mu} \bar{P}_{i, i-1,0}(s)+\frac{2 \mu(1-\gamma)}{s+\lambda+\mu} \bar{P}_{i, i-2,2}(s)\right\} ; i \geq 2 \\
\bar{P}_{i, 0,0}(s)=\frac{\mu \gamma}{s+\lambda+i \theta} \bar{P}_{i, 0,1}(s) ; i \geq 1 \\
\frac{\lambda}{s+\lambda+\mu+(i-2) \theta} \bar{P}_{i, 1,2}(s) ; i>2
\end{gathered}
$$




$$
\begin{gathered}
\bar{P}_{i, i-1,1}(s)=\frac{\lambda}{s+\lambda+\mu} \bar{P}_{i-1, i-1,0}(s)+\frac{\theta}{s+\lambda+\mu} \bar{P}_{i, i-1,0}(s)+\frac{2 \mu(1-\gamma)}{s+\lambda+\mu} \bar{P}_{i, i-2,2}(s) ; i>1 \\
\bar{P}_{i, 2,0}(s)=\frac{\mu(1-\gamma)}{s+\lambda+(i-2) \theta} \bar{P}_{i, 1,1}(s)+\frac{\mu \gamma}{s+\lambda+(i-2) \theta} \bar{P}_{i, 2,1}(s) ; i>2 \\
\bar{P}_{i, j, 2}(s)=\sum_{k=1}^{i-j}\left(\frac{\lambda}{s+\lambda+2 \mu}\right)^{i-j-k} \eta_{k}^{\prime}(s) \bar{P}_{j+k, j, 1}(s) ; i \geq j+2, j \geq 1
\end{gathered}
$$

Where

$$
\begin{gathered}
\eta_{k}^{\prime}=\left\{\begin{array}{cc}
1 & k=1 \\
1+\frac{(k-1) \theta}{s+\lambda+2 \mu} & k=2 \text { to } i-j-1 \\
\frac{(k-1) \theta}{s+\lambda+2 \mu} & k=i-j
\end{array}\right. \\
\bar{P}_{i, j, 1}(s)=\frac{\lambda}{s+\lambda+\mu+(i-j-1) \theta} \bar{P}_{i-1, j, 0}(s)+\frac{(i-j) \theta}{s+\lambda+\mu+(i-j-1) \theta} \bar{P}_{i, j, 0}(s)+ \\
\frac{2 \mu(1-\gamma)}{s+\lambda+\mu+(i-j-1) \theta} \sum_{k=1}^{i-j}\left(\frac{\lambda}{s+\lambda+2 \mu}\right)^{i-j-k} \eta_{k}^{\prime}(s) \bar{P}_{j+k-1, j-1,1}(s)+ \\
\frac{2 \mu \gamma}{s+\lambda+\mu+(i-j-1) \theta} \sum_{k=1}^{i-j}\left(\frac{\lambda}{s+\lambda+2 \mu}\right)^{i-j-k} \eta_{k}^{\prime}(s) \bar{P}_{j+k, j, 1}(s) ; \quad i \geq j+2, j \geq 2
\end{gathered}
$$

where

$$
\begin{gathered}
\eta_{k}^{\prime}=\left\{\begin{array}{cl}
1+\frac{(k-1) \theta}{s+\lambda+2 \mu} & k=1 \\
\frac{(k-1) \theta}{s+\lambda+2 \mu} & k=2 \text { to } i-j-1 \\
\frac{1}{s+\lambda} & k=i-j
\end{array}\right. \\
\bar{P}_{i, j, 0}(s)=\frac{\mu(1-\gamma)}{s+\lambda+(i-j) \theta} \bar{P}_{i, j-1,1}(s)+\frac{\mu \gamma}{s+\lambda+(i-j) \theta} \bar{P}_{i, j, 1}(s)
\end{gathered}
$$

Taking the Inverse Laplace of equations (7) to (17)

$$
\begin{gathered}
P_{0,0,0}(t)=e^{-\lambda t} \\
P_{1,0,1}(t)=\lambda e^{-(\lambda+\mu) t} * P_{0,0,0}(t)+\theta e^{-(\lambda+\mu) t} * P_{1,0,0}(t) \\
P_{1,1,0}(t)=\mu(1-\gamma) e^{-\lambda t} * P_{1,0,1}(t) \\
P_{i, 0,0}(t)=\mu \gamma e^{-(\lambda+i \theta) t} * P_{i, 0,1}(t) ; i \geq 1 \\
P_{i, i, 0}(t)=\mu(1-\gamma) \lambda e^{-\lambda t}\left\{\frac{1}{\mu}-\frac{e^{-\mu t}}{\mu}\right\} * P_{i-1, i-1,0}(t)+\mu(1-\gamma) \theta e^{-\lambda t}\left\{\frac{1}{\mu}-\frac{e^{-\mu t}}{\mu}\right\} * \\
P_{i, i-1,0}(t)+2 \mu^{2}(1-\gamma)^{2} e^{-\lambda t}\left\{\frac{1}{\mu}-\frac{e^{-\mu t}}{\mu}\right\} * P_{i, i-2,2}(t) ; \quad i \geq 2
\end{gathered}
$$




$$
\begin{aligned}
& P_{i, 1,1}(t)=\lambda e^{-(\lambda+\mu+(i-2) \theta) t} * P_{i-1,1,0}(t)+(i-1) \theta e^{-(\lambda+\mu+(i-2) \theta) t} * P_{i, 1,0}(t)+ \\
& 2 \mu(1-\gamma) e^{-(\lambda+\mu+(i-2) \theta) t} * P_{i, 0,2}(t)+2 \mu \gamma e^{-(\lambda+\mu+(i-2) \theta) t} * P_{i, 1,2}(t) ; i>2 \\
& P_{i, i-1,1}(t)=\lambda e^{-(\lambda+\mu) t} * P_{i-1, i-1,0}(t)+\theta e^{-(\lambda+\mu) t} * P_{i, i-1,0}(t)+2 \mu(1-\gamma) e^{-(\lambda+\mu) t} * P_{i, i-2,2}(t) ; i \geq 1 \\
& P_{i, 2,0}(t)=\mu(1-\gamma) e^{-(\lambda+\mu+(i-2) \theta) t} * P_{i, 1,1}(t)+\mu \gamma e^{-(\lambda+\mu+(i-2) \theta) t} * P_{i, 2,1}(t) ; i>2 \\
& P_{i, j, 2}(t)=\lambda^{i-j-1} \frac{t^{i-j}}{(i-j) !} e^{-(\lambda+2 \mu) t} * P_{j+1, j, 1}(t)+\sum_{k=2}^{i-j-1} \lambda^{i-j-k} \frac{t^{i-j-k-1}}{(i-j-k-1) !} e^{-(\lambda+2 \mu) t} * P_{j+k, j, 1}(t)+ \\
& \sum_{k=2}^{i-j-1} \lambda^{i-j-k}(k-1) \theta \frac{t^{i-j-k}}{(i-j-k) !} e^{-(\lambda+2 \mu) t} * P_{j+k, j, 1}(t)+(i-j-1) \theta e^{-(\lambda+2 \mu) t} * P_{i, j, 1}(t) ; i \geq j+2, j \geq 1 \\
& P_{i, j, 1}(t)=\lambda e^{-(\lambda+\mu+(i-j-1) \theta) t} * P_{i-1, j, 0}(t)+(i-j) \theta e^{-(\lambda+\mu+(i-j-1) \theta) t} * P_{i, j, 0}(t)+ \\
& 2 \mu(1-\gamma) \lambda^{i-j-1} e^{-(\lambda+\mu+(i-j-1) \theta) t}\left\{\frac{1}{2 \mu}-e^{-2 \mu t} \sum_{r=0}^{i-j-2} \frac{t^{r}}{r !} \frac{1}{(2 \mu)^{i-j-1-r}}\right\} * \\
& P_{j, j-1,1}(t)+2 \mu(1-\gamma) e^{-(\lambda+\mu+(i-j-1) \theta) t} \sum_{k=2}^{i-j-1} \lambda^{i-j-k}\left\{\frac{1}{(2 \mu)^{i-j-k}}-\right. \\
& \left.e^{-2 \mu t} \sum_{r=0}^{i-j-k-1} \frac{t^{r}}{r !} \frac{1}{(2 \mu)^{i-j-k-r}}\right\} * P_{j+k-1, j-1,1}(t)+2 \mu(1- \\
& \text { r) } e^{-(\lambda+\mu+(i-j-1) \theta) t} \sum_{k=2}^{i-j-1} \lambda^{i-j-k}(k-1) \theta\left\{\frac{1}{(2 \mu)^{i-j-k}}-\right. \\
& \left.e^{-2 \mu t} \sum_{r=0}^{i-j-k-1} \frac{t^{r}}{r !} \frac{1}{(2 \mu)^{i-j-k-r}}\right\} * P_{j+k-1, j-1,1}(t)+2 \mu(1-\gamma)(i-j-1) \\
& \theta e^{-(\lambda+\mu+(i-j-1) \theta) t}\left\{\frac{1}{2 \mu}-\frac{e^{-2 \mu t}}{2 \mu}\right\} * P_{i, j, 1}(t)+2 \mu \gamma e^{-(\lambda+\mu+(i-j-1) \theta) t} \lambda^{i-j-1}\left\{\frac{1}{2 \mu}-\right. \\
& \left.e^{-\lambda t} \sum_{r=0}^{i-j-2} \frac{t^{r}}{r !} \frac{1}{(2 \mu)^{i-j-1-r}}\right\} * P_{j+1, j, 1}(t)+ \\
& 2 \mu \gamma e^{-(\lambda+\mu+(i-j-1) \theta) t} \sum_{k=2}^{i-j-1} \lambda^{i-j-k}\left\{\frac{1}{(2 \mu)^{i-j-k}}-e^{-2 \mu t} \sum_{r=0}^{i-j-k-1} \frac{t^{r}}{r !} \frac{1}{(2 \mu)^{i-j-k-r}}\right\} * \\
& P_{j+k, j, 1}(t)+2 \mu \gamma e^{-(\lambda+\mu+(i-j-1) \theta) t} \sum_{k=2}^{i-j-1} \lambda^{i-j-k}(k-1) \theta\left\{\frac{1}{(2 \mu)^{i-j-k}}-\right. \\
& \left.e^{-2 \mu t} \sum_{r=0}^{i-j-k-1} \frac{t^{r}}{r !} \frac{1}{(2 \mu)^{i-j-k-r}}\right\} * P_{j+k, j, 1}(t)+2 \mu \gamma e^{-(\lambda+\mu+(i-j-1) \theta) t}(i-j-1) \theta\left\{\frac{1}{2 \mu}-\right. \\
& \left.\frac{e^{-2 \mu t}}{2 \mu}\right\} * P_{i, j, 1}(t) ; \quad i \geq j+2, j \geq 2 \\
& P_{i, j, 0}(t)=\mu(1-\gamma) e^{-(\lambda+(i-j) \theta) t} * P_{i, j-1,1}(t)+\mu \gamma e^{-(\lambda+(i-j) \theta) t} * P_{i, j, 1}(t)
\end{aligned}
$$

\section{Some Important Performance Measures}

- The Laplace transform of $\bar{P}_{i .}(s)$ is given as:

$$
\bar{P}_{i .}(s)=\sum_{j=0}^{i} \bar{P}_{i, j}(s)=\frac{\lambda^{i}}{(s+\lambda)^{i+1}} ; i>0
$$


and its Inverse Laplace is:

$$
P_{i .}(t)=\frac{e^{-\lambda t}(\lambda t)^{i}}{i !}
$$

which proves the basic assumption that primary arrivals follow Poisson process.

- The probability that exactly $\mathrm{j}$ customers depart from the system by time $t$ is given as:

$$
P_{. j}(t)=\sum_{i=j}^{\infty} P_{i, j}(t)
$$

- Summing equations (7)-(17) over $i$ and $j$ we get:

$$
\sum_{i=0}^{\infty} \sum_{j=0}^{i}\left\{\bar{P}_{i, j, 0}(s)+\bar{P}_{i, j, 1}(s)+\bar{P}_{i, j, 2}(s)\right\}=\frac{1}{s}
$$

and hence

$$
\sum_{i=0}^{\infty} \sum_{j=0}^{i}\left\{P_{i, j, 0}(t)+P_{i, j, 1}(t)+P_{i, j, 2}(t)\right\}=1
$$

which is the verification of our results.

- Define $Q_{n, m}=$ Probabilitiy that there are n customers in the orbit when $\mathrm{m}(\mathrm{m}=0,1,2)$ servers are busy at time t.

When server is free, it is represented by probability $Q_{n, 0}(t)$

$$
Q_{n, 0}(t)=\sum_{j=0}^{\infty} P_{j+n, j, 0}(t)
$$

The number of customers in the orbit, in this case are calculated with the following formula:

$$
n=(\text { number of arrivals }- \text { number of departures) }
$$

When $\mathrm{m}$ servers are busy, it is represented by the probability $Q_{n, m}(\mathrm{t})$.

$$
Q_{n, m}(t)=\sum_{j=0}^{\infty} P_{j+n+m, j, m}(t) ; \quad m=1,2
$$

The number of customers in the orbit in this case is calculated by the following formula:

$$
n=(\text { number of arrivals }- \text { number of departures }-m)
$$

Using above definitions in (1) to (3) and let $\mu=1, \gamma=0$ the equations we get are:

$$
\begin{gathered}
(\lambda+n \theta) Q_{n, 0}(t)=Q_{n, 1}(t) ; n \geq 0 \\
(\lambda+n \theta+1) Q_{n, 1}(t)=\lambda Q_{n, 0}(t)+(n+1) \theta Q_{n+1,0}(t)+2 Q_{n, 2}(t) ; n \geq 0 \\
(\lambda+2) Q_{n, 2}(t)=\lambda Q_{n, 1}(t)+\lambda Q_{n-1,2}(t)+(n+1) \theta Q_{n+1,1}(t) ; n \geq 0
\end{gathered}
$$

which coincides with the results (1)-(3) of ${ }^{(17)}$.

\section{Numerical Solution and Graphical Representation}

The Numerical solutions are generated using MATLAB programming for the case $\rho=0.6, \eta=0.5$ and $\gamma=0.4$. Observing the below tables for various time instants it could be seen that the sum of probabilities approaches to 1 . 
Table 1. At $\mathrm{t}=1$

\begin{tabular}{llllllllll}
\hline$P_{0,0,0}$ & $P_{1,0,0}$ & $P_{1,1,0}$ & $P_{2,0,0}$ & $P_{1,0,1}$ & $P_{2,0,1}$ & $P_{2,1,1}$ & $P_{2,0,2}$ & $P_{3,0,2}$ & Sum \\
\hline 0.5488 & 0.0414 & 0.0737 & 0.0016 & 0.2142 & 0.0162 & 0.0288 & 0.0418 & 0.0085 & 0.975 \\
\hline
\end{tabular}

Table 2. At $\mathrm{t}=5$

\begin{tabular}{llllllllll}
\hline$P_{0,0,0}$ & $P_{1,0,0}$ & $P_{1,1,0}$ & $P_{2,1,0}$ & $P_{2,2,0}$ & $P_{3,2,0}$ & $P_{3,3,0}$ & $P_{4,3,0}$ & $P_{5,3,0}$ & $P_{5,4,0}$ \\
\hline 0.0498 & 0.0249 & 0.0845 & 0.0423 & 0.0717 & 0.0366 & 0.0378 & 0.0198 & 0.0153 & 0.0177 \\
\hline$P_{1,0,1}$ & $P_{2,0,1}$ & $P_{2,1,1}$ & $P_{3,1,1}$ & $P_{3,2,1}$ & $P_{4,1,1}$ & $P_{4,2,1}$ & $P_{4,3,1}$ & $P_{5,2,1}$ & $P_{5,3,1}$ \\
\hline 0.0399 & 0.02 & 0.0678 & 0.0367 & 0.0551 & 0.0139 & 0.0139 & 0.0315 & 0.0226 & 0.0301 \\
\hline & $P_{5,4,1}$ & $P_{2,0,2}$ & $P_{3,1,2}$ & $P_{4,1,2}$ & $P_{4,2,2}$ & $P_{5,1,2}$ & $P_{5,2,2}$ & $P_{5,3,2}$ & Sum \\
\hline & 0.0171 & 0.016 & 0.0243 & 0.0198 & 0.0177 & 0.0155 & 0.0221 & 0.0121 & 0.8477 \\
\hline
\end{tabular}

Table 3. At $\mathrm{t}=10$

\begin{tabular}{llllllllll}
\hline$P_{5,3,0}$ & $P_{5,4,0}$ & $P_{5,5,0}$ & $P_{2,1,1}$ & $P_{3,1,1}$ & $P_{3,2,1}$ & $P_{4,2,1}$ & $P_{4,3,1}$ & $P_{5,2,1}$ & $P_{5,3,1}$ \\
\hline 0.0489 & 0.1496 & 0.2153 & 0.0101 & 0.0075 & 0.0219 & 0.0169 & 0.0307 & 0.0254 & 0.0798 \\
\hline & $P_{5,4,1}$ & $P_{3,1,2}$ & $P_{4,2,2}$ & $P_{5,2,2}$ & $P_{5,3,2}$ & Sum & & \\
\hline & 0.1187 & 0.0045 & 0.0094 & 0.023 & 0.0302 & 0.9387 & \\
\hline
\end{tabular}

Table 4. At $\mathrm{t}=15$

\begin{tabular}{llllllllll}
\hline$P_{2,2,0}$ & $P_{3,2,0}$ & $P_{3,3,0}$ & $P_{4,3,0}$ & $P_{4,4,0}$ & $P_{5,3,0}$ & $P_{5,4,0}$ & $P_{5,5,0}$ & $P_{3,2,1}$ & $P_{4,3,1}$ \\
\hline 0.0032 & 0.0025 & 0.0078 & 0.0061 & 0.0138 & 0.0224 & 0.1566 & 0.5927 & 0.0032 & 0.0076 \\
\hline & $P_{5,3,1}$ & $P_{5,4,1}$ & $P_{2,0,2}$ & $P_{4,2,2}$ & $P_{5,2,2}$ & $P_{5,3,2}$ & Sum & \\
\hline & 0.0338 & 0.1123 & 0.0001 & 0.0015 & 0.023 & 0.0302 & 0.9809 \\
\hline
\end{tabular}

Table 5. At $\mathrm{t}=25$

\begin{tabular}{llllllllll}
\hline$P_{0,0,0}$ & $P_{1,1,0}$ & $P_{2,2,0}$ & $P_{3,1,0}$ & $P_{3,3,0}$ & $P_{4,3,0}$ & $P_{4,4,0}$ & $P_{5,4,0}$ & $P_{5,5,0}$ & $P_{1,0,1}$ \\
\hline 0 & 0 & 0 & 0 & 0.0001 & 0.0001 & 0.0004 & 0.0303 & 0.9462 & 0 \\
\hline & $P_{2,1,1}$ & $P_{3,1,1}$ & $P_{4,2,1}$ & $P_{4,3,1}$ & $P_{2,0,2}$ & $P_{3,1,2}$ & $P_{4,2,2}$ & $P_{5,2,2}$ & Sum \\
\hline & 0 & 0 & 0 & 0.0001 & 0 & 0 & 0 & 0.0001 & 0.9773 \\
\hline
\end{tabular}

Table 6. At $\mathrm{t}=35$

\begin{tabular}{llllllllllll}
\hline$P_{0,0,0}$ & $P_{1,1,0}$ & $P_{2,1,0}$ & $P_{3,2,0}$ & $P_{4,2,0}$ & $P_{4,4,0}$ & $P_{5,3,0}$ & $P_{5,5,0}$ & $P_{1,0,1}$ & $P_{2,1,1}$ & $P_{3,2,1}$ & $P_{4,1,1}$ \\
\hline 0 & 0 & 0 & 0 & 0 & 0 & 0 & 0.9947 & 0 & 0 & 0 & 0 \\
\hline & $P_{5,0,1}$ & $P_{5,2,1}$ & $P_{5,4,1}$ & $P_{2,0,2}$ & $P_{3,1,2}$ & $P_{4,2,2}$ & $P_{5,3,2}$ & Sum & & \\
\hline & 0 & 0 & 0.0021 & 0 & 0 & 0 & 0 & 0.9968 & \\
\hline
\end{tabular}

Table 7. At $\mathrm{t}=40$

\begin{tabular}{lllllllllll}
\hline$P_{0,0,0}$ & $P_{1,1,0}$ & $P_{2,2,0}$ & $P_{3,1,0}$ & $P_{3,3,0}$ & $P_{4,1,0}$ & $P_{5,0,0}$ & $P_{5,5,0}$ & $P_{1,0,1}$ & $P_{3,1,1}$ & $P_{3,2,1}$ \\
\hline 0 & 0 & 0 & 0 & 0 & 0 & 0 & 0.9984 & 0 & 0 & 0 \\
\hline & $P_{4,1,1}$ & $P_{4,3,1}$ & $P_{5,3,1}$ & $P_{5,4,1}$ & $P_{3,1,2}$ & $P_{4,0,2}$ & $P_{5,1,2}$ & $P_{5,3,2}$ & Sum \\
\hline & 0 & 0 & 0 & 0.0006 & 0 & 0 & 0 & 0 & 0.999 & \\
\hline
\end{tabular}


The probabilities against time are presented graphically through Figures 3, 4, 5, 6, 7, 8, 9 and 10.

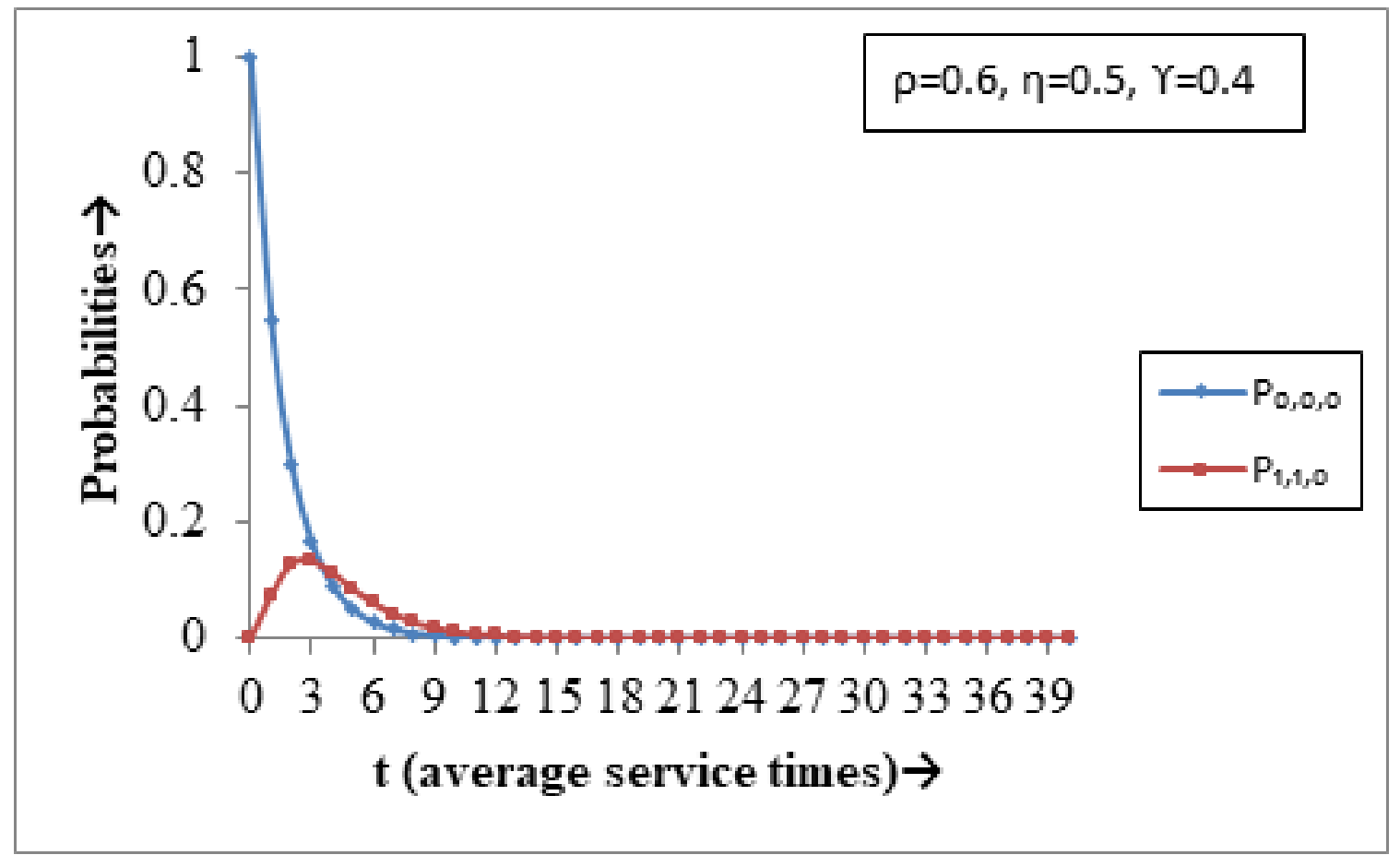

Fig 3. Probabilities $P_{0,0,0}$ and $P_{1,1,0}$ against t (average service times)

Figure 3 depicts the plot of probabilities $P_{0,0,0}$ and $P_{1,1,0}$ against time $\mathrm{t}$ (average service times) for the case when $\rho=0.6, \eta$ $=0.5$ and $\gamma=0.4$. It is clear from the graph that the probability $P_{0,0,0}$ rapidly decreases with time from initial value 1 at $\mathrm{t}=0$. The probability $P_{1,1,0}$ increases in the beginning from initial value 0 at $\mathrm{t}=0$ and then decreases gradually.

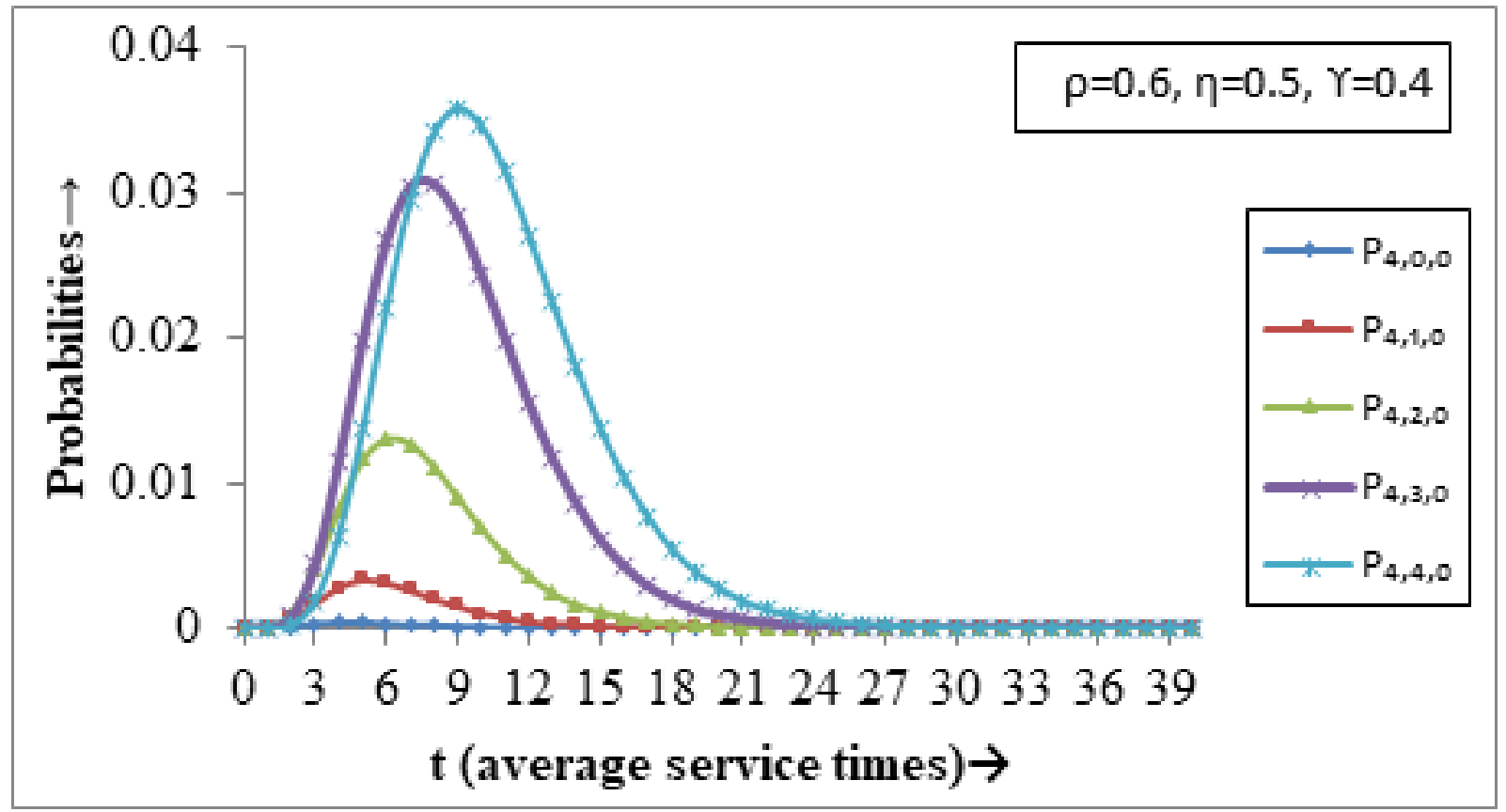

Fig 4. Probabilities $P_{4,1,0} P_{4,2,0} P_{4,3,0} P_{4,4,0} P_{4,4,0}$ against t (average service times) 
Figure 4 depicts comparison among the probabilities $P_{4,0,0}, P_{4,1,0}, P_{4,2,0}, P_{4,3,0}$ and $P_{4,4,0}$ for the case when $\rho=0.6, \eta=0.5$ and $\gamma=0.4$. It is interpreted from the graph that the highest values attained are by probabilities are larger for higher number of departures.

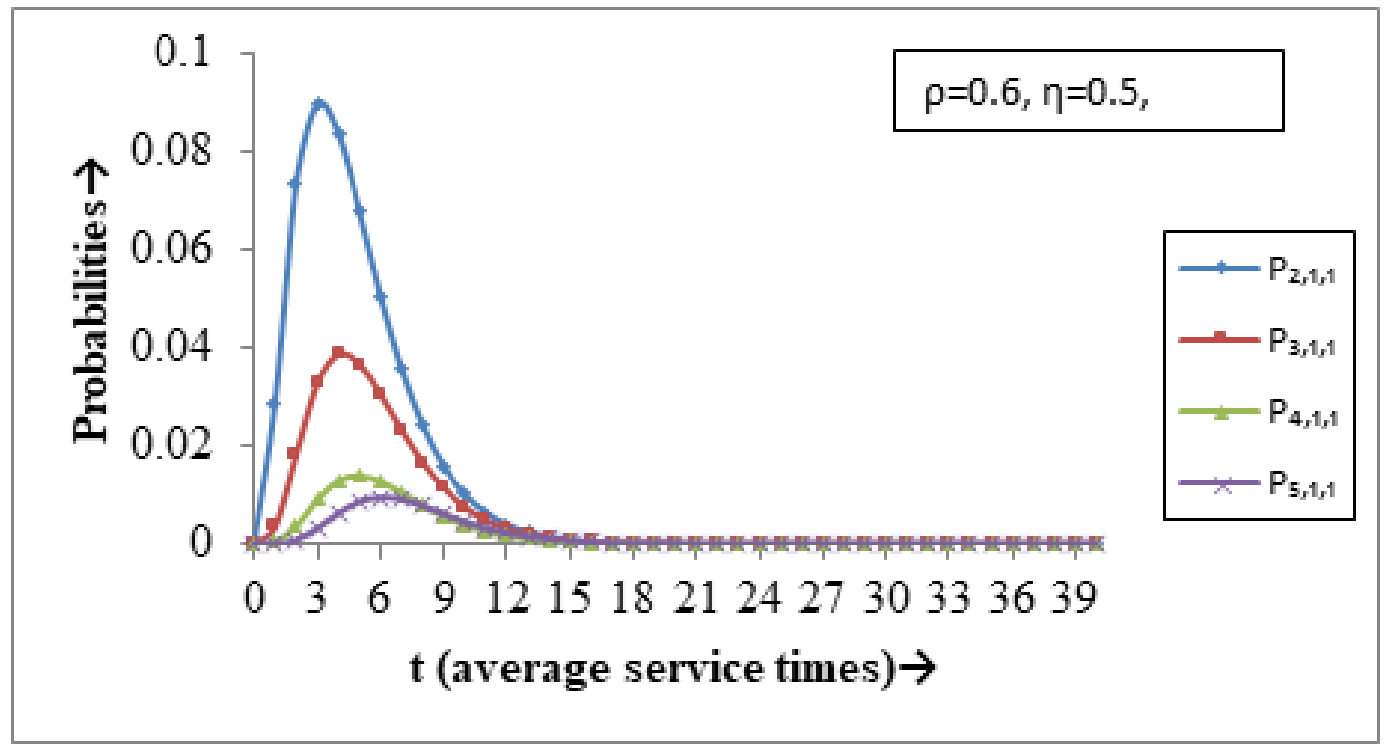

Fig 5. Probabilities $P_{2,1,1} P_{3,1,1} P_{4,1,1} P_{5,1,1}$ against t (average service times)

The probabilities $P_{2,1,1}, P_{3,1,1}, P_{4,1,1}$ and $P_{5,1,1}$ for the case when $\rho=0.6, \eta=0.5$ and $\gamma=0.4$ are compared in Figure 5. It is interpreted from the graph that in the starting the probabilities increases and then decreases. In general, it can also be concluded that the probabilities $P_{i, 0,1}$ take smaller values for higher i (number of arrivals).

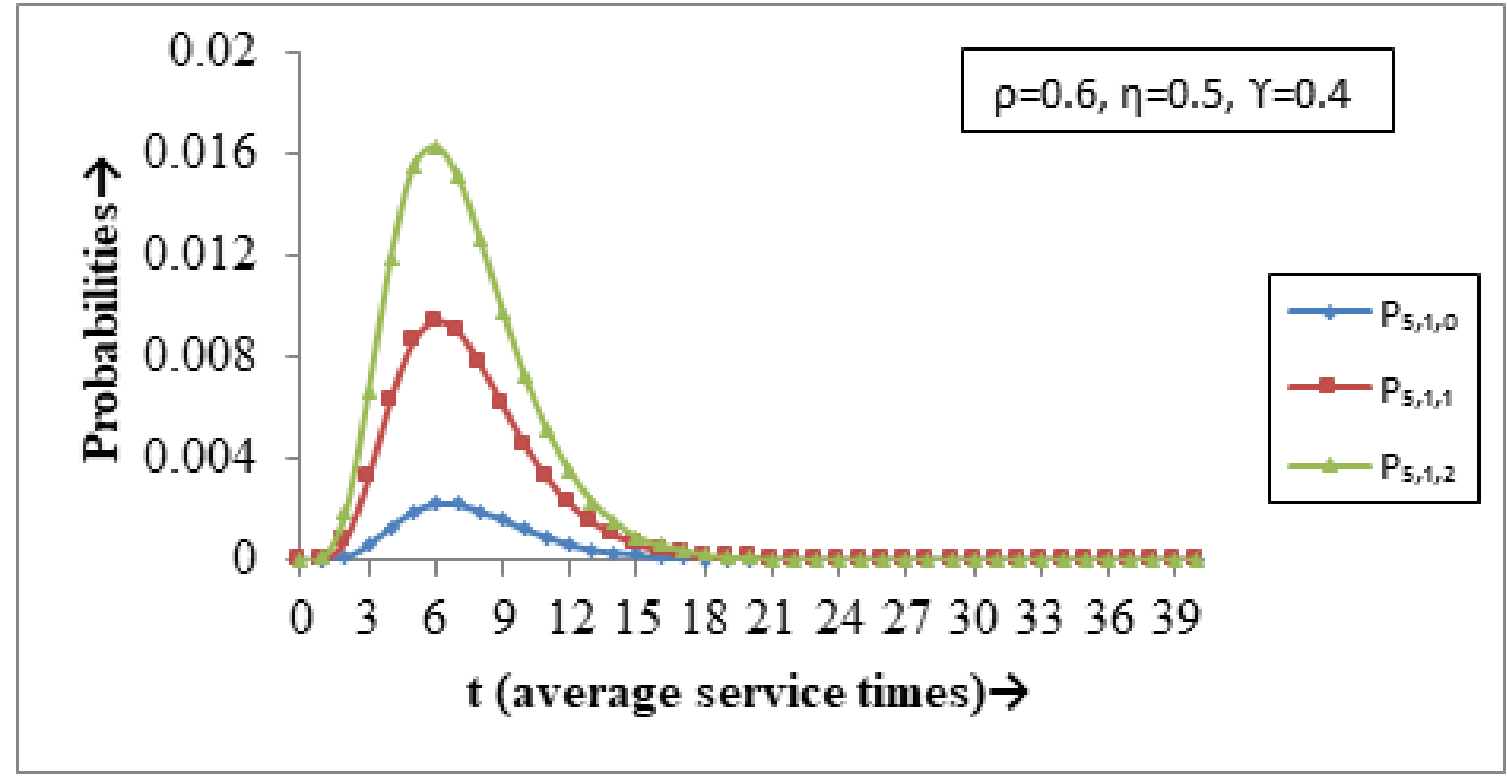

Fig 6. Probabilities $P_{5,1,0} P_{5,1,1} P_{5,1,2} P_{5,1,1}$ against t (average service times)

Figure 6 shows the comparison between probabilities $P_{5,1,0}, P_{5,1,1}$ and $P_{5,1,2}$ when plotted against time $\mathrm{t}$ for $\rho=0.6, \eta=0.5$ and $\gamma=0.4$. Beginning with value 0 at $\mathrm{t}=0$ the probabilities $P_{5,1,0}, P_{5,1,1}$ and $P_{5,1,2}$ increases rapidly to their highest values and then decreases. It can be seen that the probability $P_{5,1,2}$ is greater than $P_{5,1,1}$ which is greater than $P_{5,1,0}$ throughout i.e., the probability when both the servers are busy is greater than probability when one server is busy which is in turn greater than the 
probability when both the servers are idle.

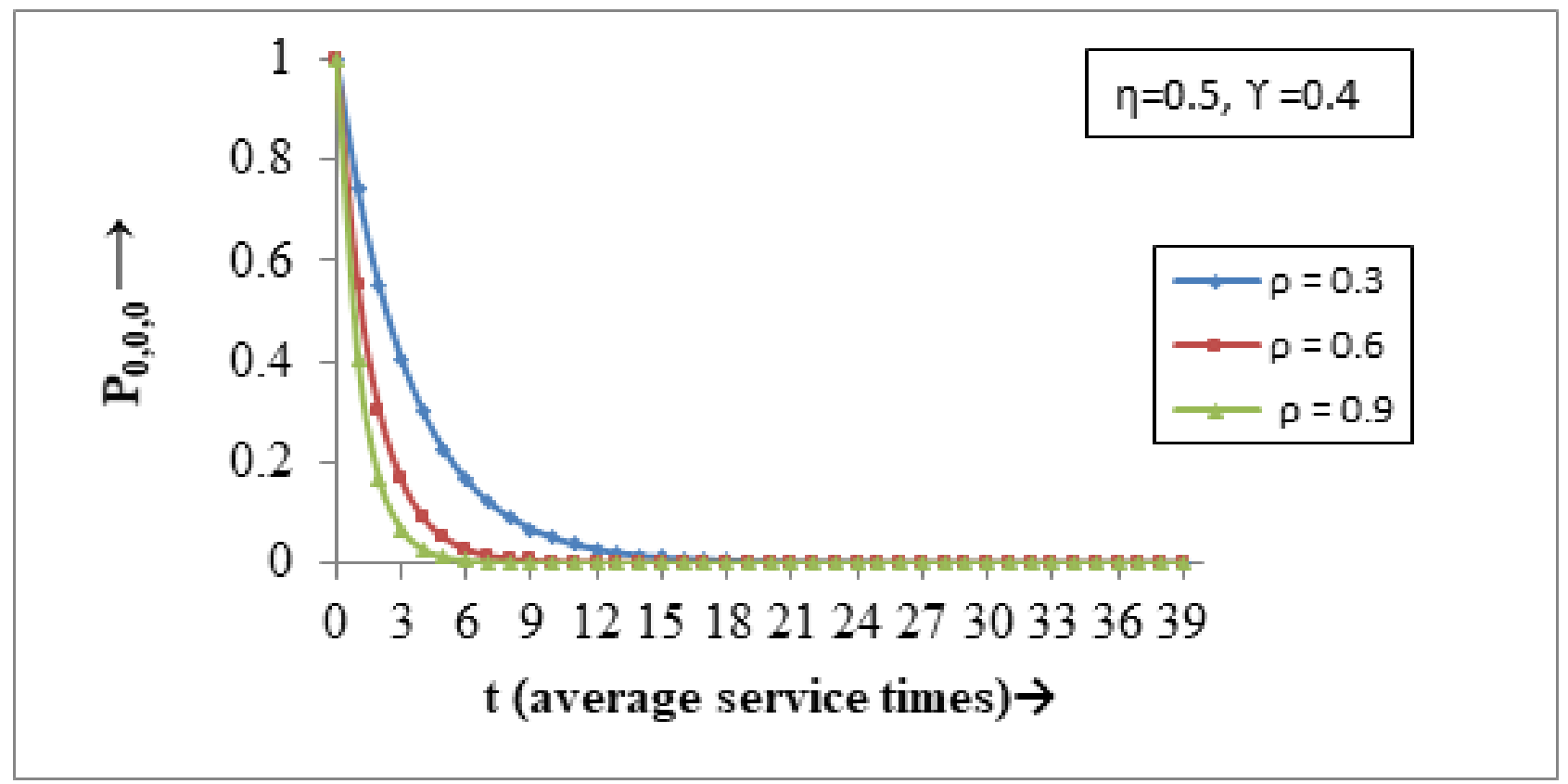

Fig 7. Effect of $\rho$ on probability $P_{0,0,0}$ against t (average service times)

The effect of change of $\rho$ (traffic intensity) on probability $P_{0,0,0}$ is studied in Figure 7 keeping other parameters constant i.e., $\eta=0.5$ and $\gamma=0.4$. The plot depicts the probability $P_{0,0,0}$ against time $t$ for different values of traffic intensity $(\rho=0.3,0.6$ and 0.9$)$ and it is concluded that for large value of $\rho$, probability $P_{0,0,0}$ decreases. Therefore, more is value of $\rho$ i.e., more are the arriving customers per unit service time, less is the probability of having zero units in the system.

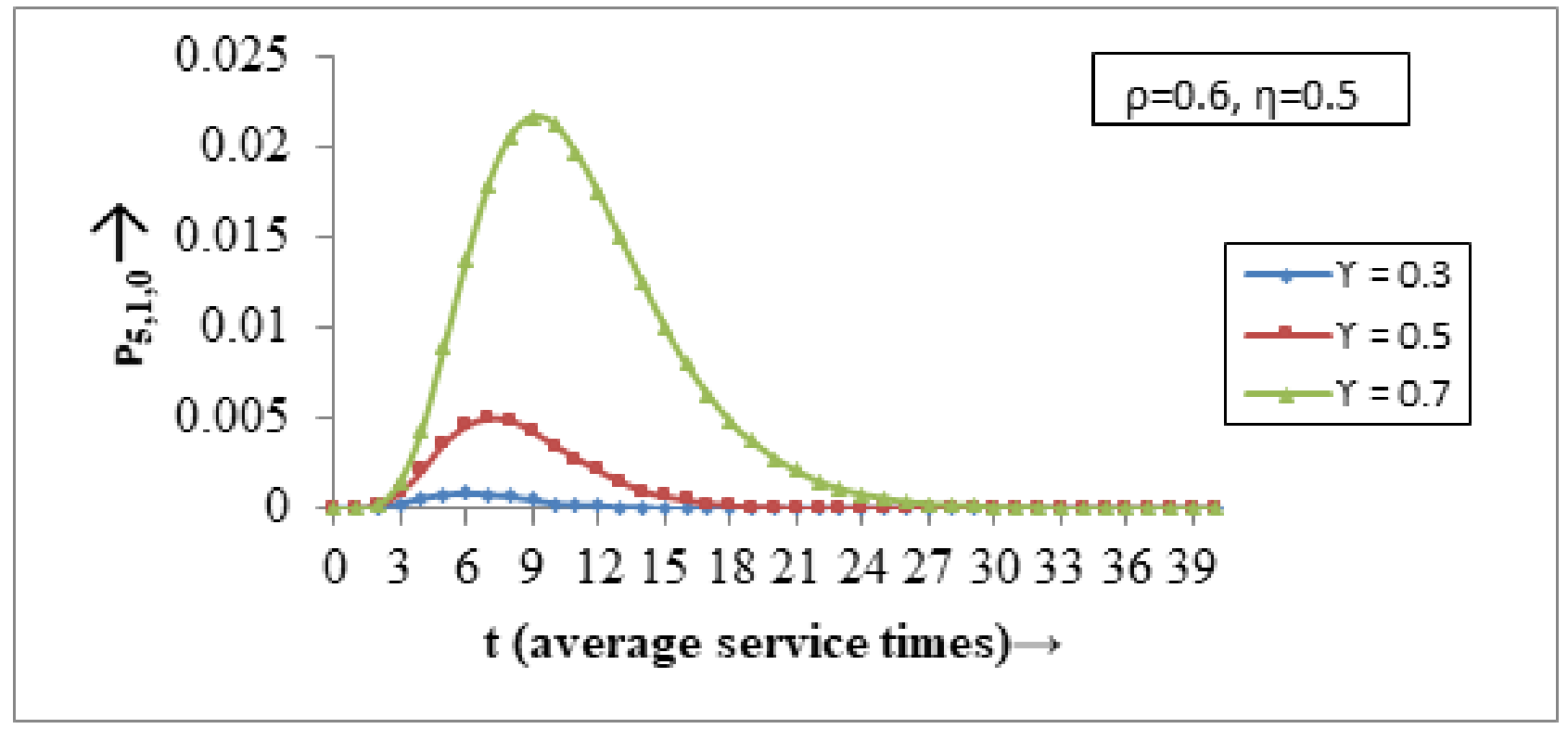

Fig 8. Effect of $\gamma$ on probability $P_{5,1,0}$, against t (average service times) 


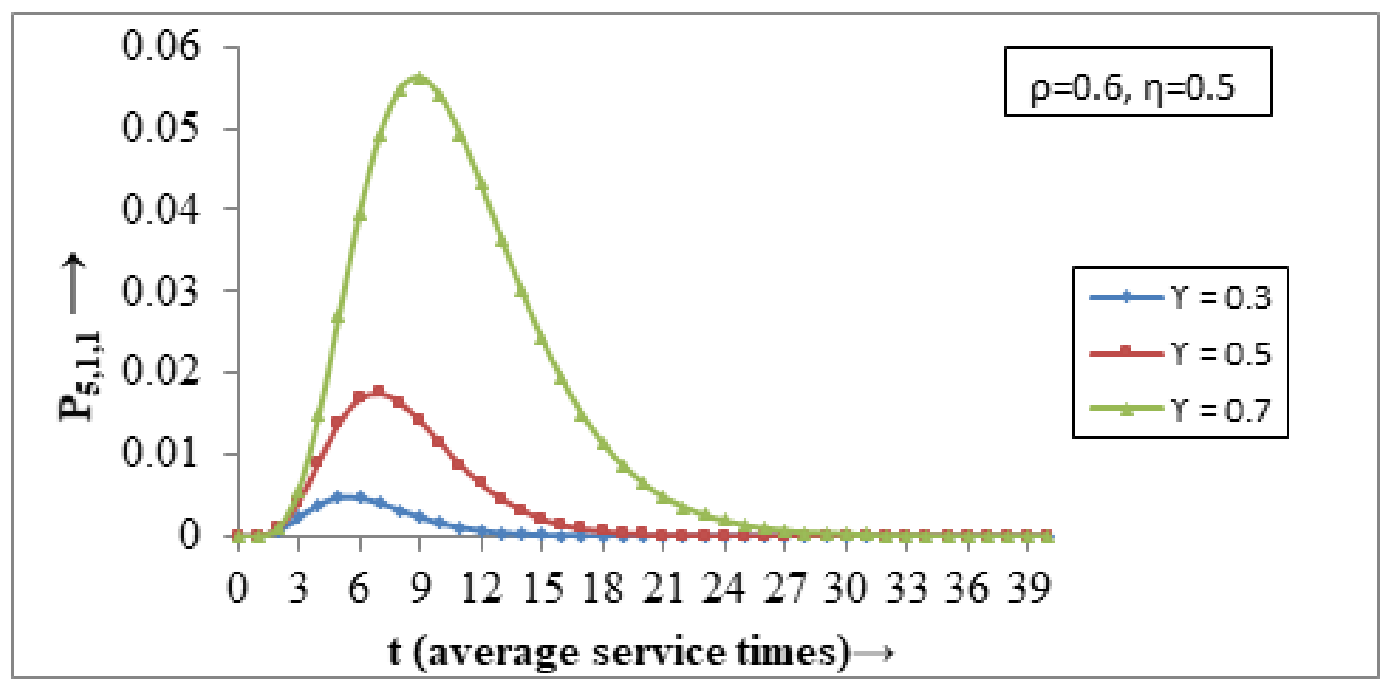

Fig 9. Effect of $\gamma$ on probability $P_{5,1,1}$, against t (average service times)

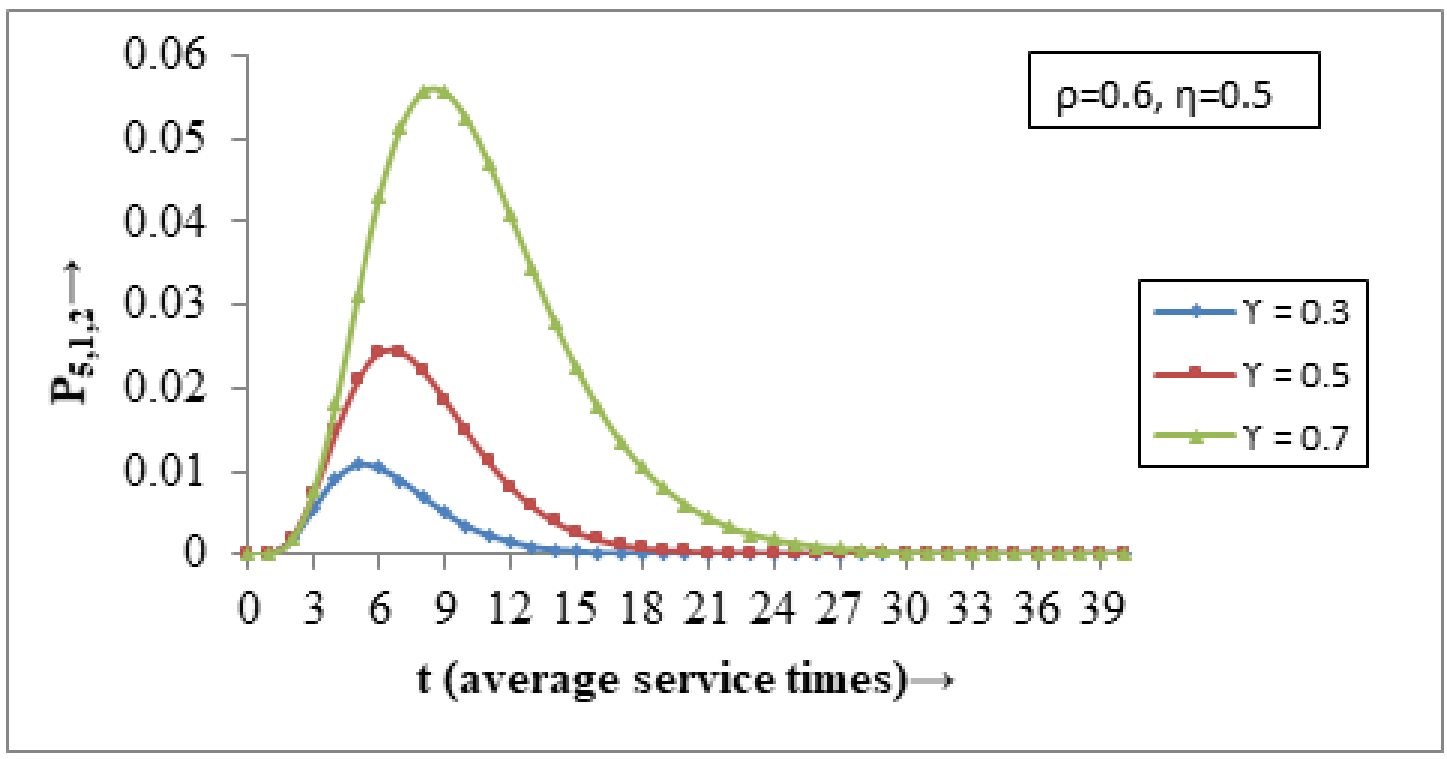

Fig 10. Effect of $\gamma$ onprobability $P_{5,1,2}$, against $\mathrm{t}$ (average service times)

In Figures 8, 9 and 10 the probabilities $P_{5,1,0}, P_{5,1,1}$, and $P_{5,1,2}$ are plotted respectively against time $\mathrm{t}$ for different values of $\gamma=0.3,0.5$ and 0.7 keeping other parameters constant i.e., $\rho=0.6$ and $\eta=0.5$. It is concluded that the probabilities $P_{5,1,0}, P_{5,1,1}$, and $P_{5,1,2}$ increase initially and then decrease for higher values of time. It is also observed that for higher value of $\gamma$ the probabilities took larger values.

\section{Busy Period Distribution}

The busy period distribution of the system as well as the server is discussed in this section.

The probability that server is busy is given by:

$$
P(\text { Server is busy })=\sum_{i>j \geq 0}\left(P_{i, j, 1}(t)+P_{i, j, 2}(t)\right)
$$


The probability that system is busy is given by:

$$
P(\text { System is busy })=\sum_{i>j \geq 0}\left(P_{i, j, 0}(t)+P_{i, j, 1}(t)+P_{i, j, 2}(t)\right)
$$

\subsection{Numerical and Graphical Representation of Busy Period}

Using MATLAB programming and following the work of ${ }^{(18)}$ the numerical results are obtained. Here the probabilities for system busy as well as server busy are obtained which are presented in the table below for various values of $\rho$ keeping $\eta$ and $\gamma$ same.

Table 8. Probabilities of System busy and Server busy to study theeffect of $\rho$

\begin{tabular}{lllllll}
\hline \multirow{2}{*}{$\mathrm{t}$} & \multicolumn{5}{l}{ Probability(System Busy) } & \multicolumn{5}{l}{ Probability(Server Busy) } \\
\cline { 2 - 7 } & $\rho=0.3$ & $\rho=0.6$ & $\rho=0.9$ & $\rho=0.3$ & $\rho=0.6$ & $\rho=0.9$ \\
\hline 0 & 0 & 0 & 0 & 0 & 0 & 0 \\
1 & 0.2077 & 0.3724 & 0.5029 & 0.1772 & 0.3227 & 0.4419 \\
2 & 0.3204 & 0.5389 & 0.6879 & 0.2475 & 0.4318 & 0.5679 \\
3 & 0.3931 & 0.6388 & 0.7807 & 0.2858 & 0.4859 & 0.6207 \\
4 & 0.444 & 0.6944 & 0.832 & 0.3111 & 0.5173 & 0.6376 \\
5 & 0.4813 & 0.7336 & 0.8541 & 0.3294 & 0.5325 & 0.6244 \\
6 & 0.5089 & 0.7554 & 0.8511 & 0.3426 & 0.5323 & 0.586 \\
7 & 0.5292 & 0.7605 & 0.8224 & 0.3515 & 0.5175 & 0.5301 \\
8 & 0.5432 & 0.749 & 0.7717 & 0.3563 & 0.4899 & 0.4652 \\
9 & 0.5516 & 0.7214 & 0.7048 & 0.3573 & 0.4523 & 0.3984 \\
10 & 0.5546 & 0.68 & 0.6283 & 0.3544 & 0.4082 & 0.3349 \\
\hline
\end{tabular}

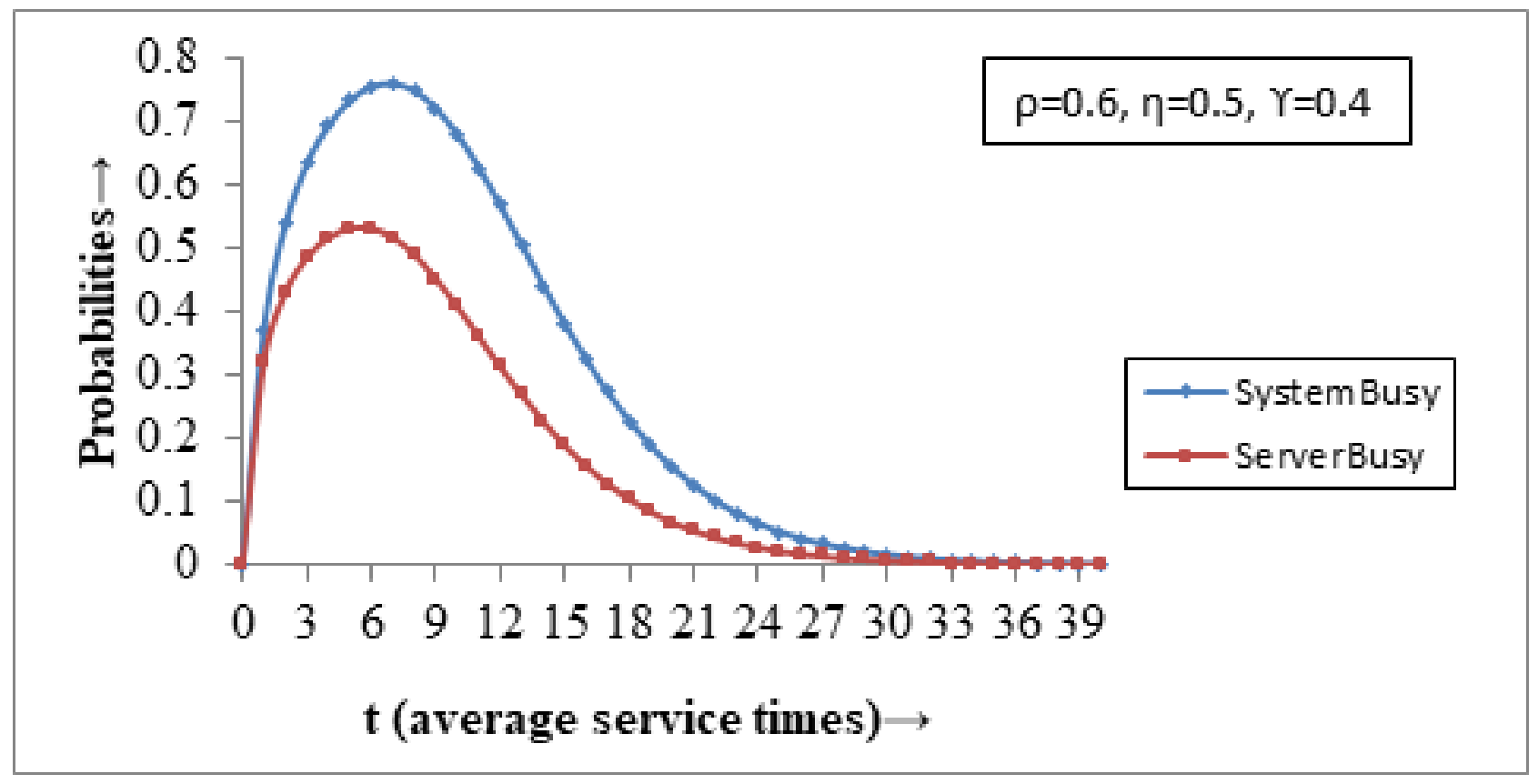

Fig 11. Probabilities of System busy and Server busy against t(average service times)

The probabilities for System busy and Server busy are plotted in Figure 11 for the case $\rho=0.6, \eta=0.5$ and $\gamma=0.4$. The two curves increase rapidly in the beginning and then start decreasing. The probability of System busy remains higher than probability of Server busy, as required. 


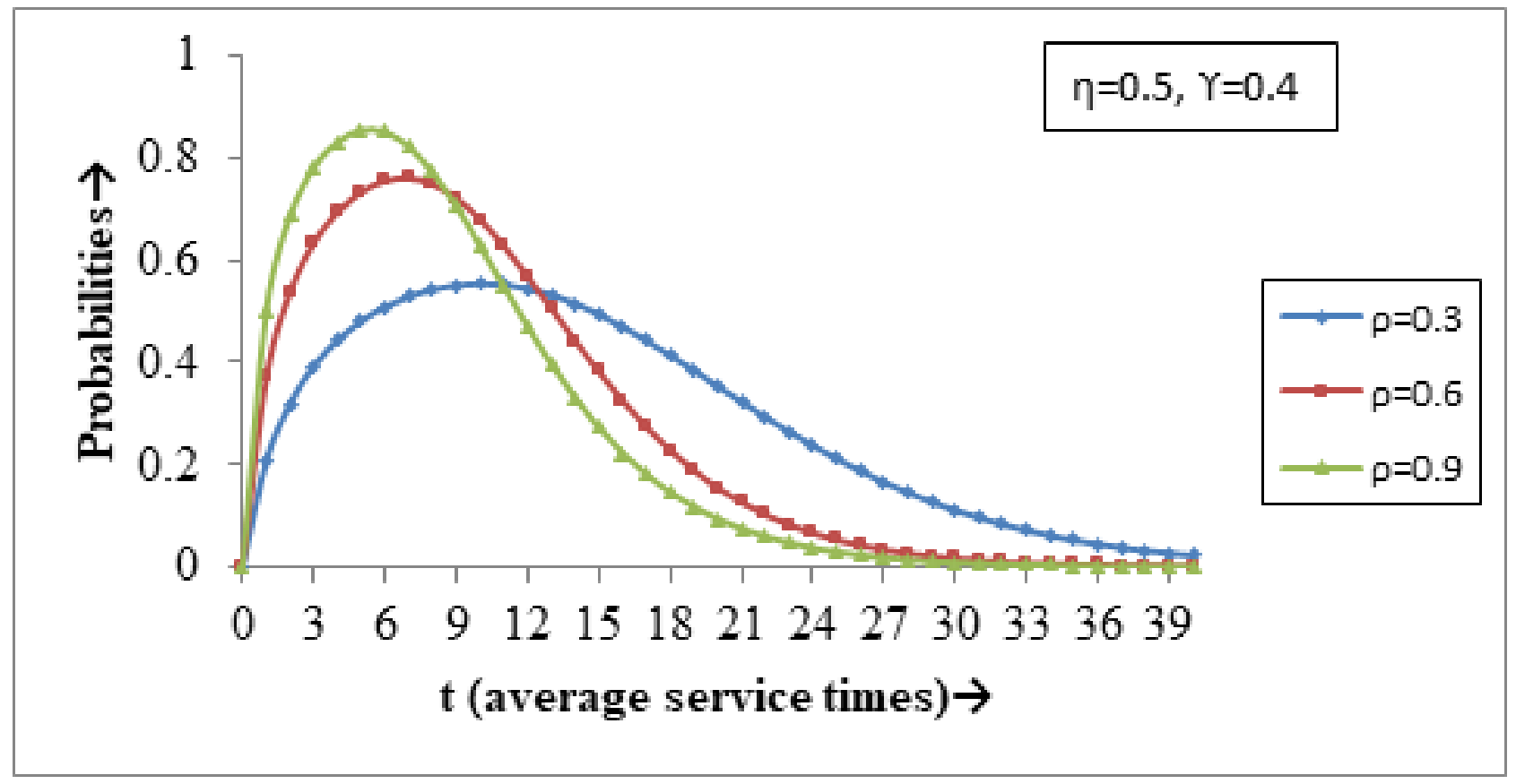

Fig 12. Effect of $\rho$ on System busy against t (average service times)

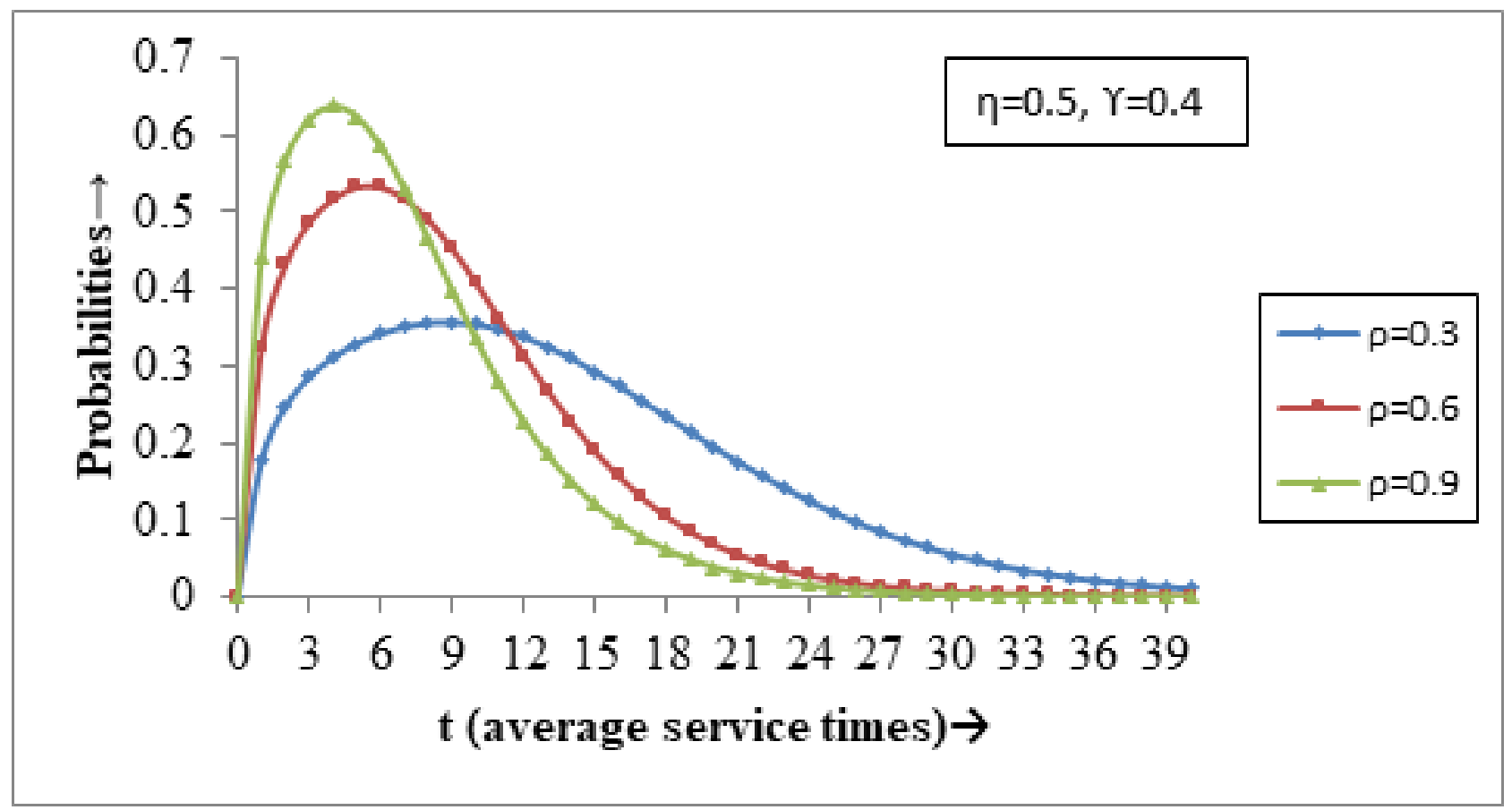

Fig 13. Effect of $\rho$ on Server busy against t (average servicetimes)

The probabilities of System busy and Server busy are plotted against time for different values of $\rho$ (traffic intensity $\left.=\frac{\lambda}{2 \mu}\right)$ in Figures 12 and 13 respectively. It is clearly visible from both the graphs that the probabilities have larger values for higher values of $\rho$ in the initial to moderate values of $t$. Afterwards this trend of these probabilities reversed for higher values of $t$. 


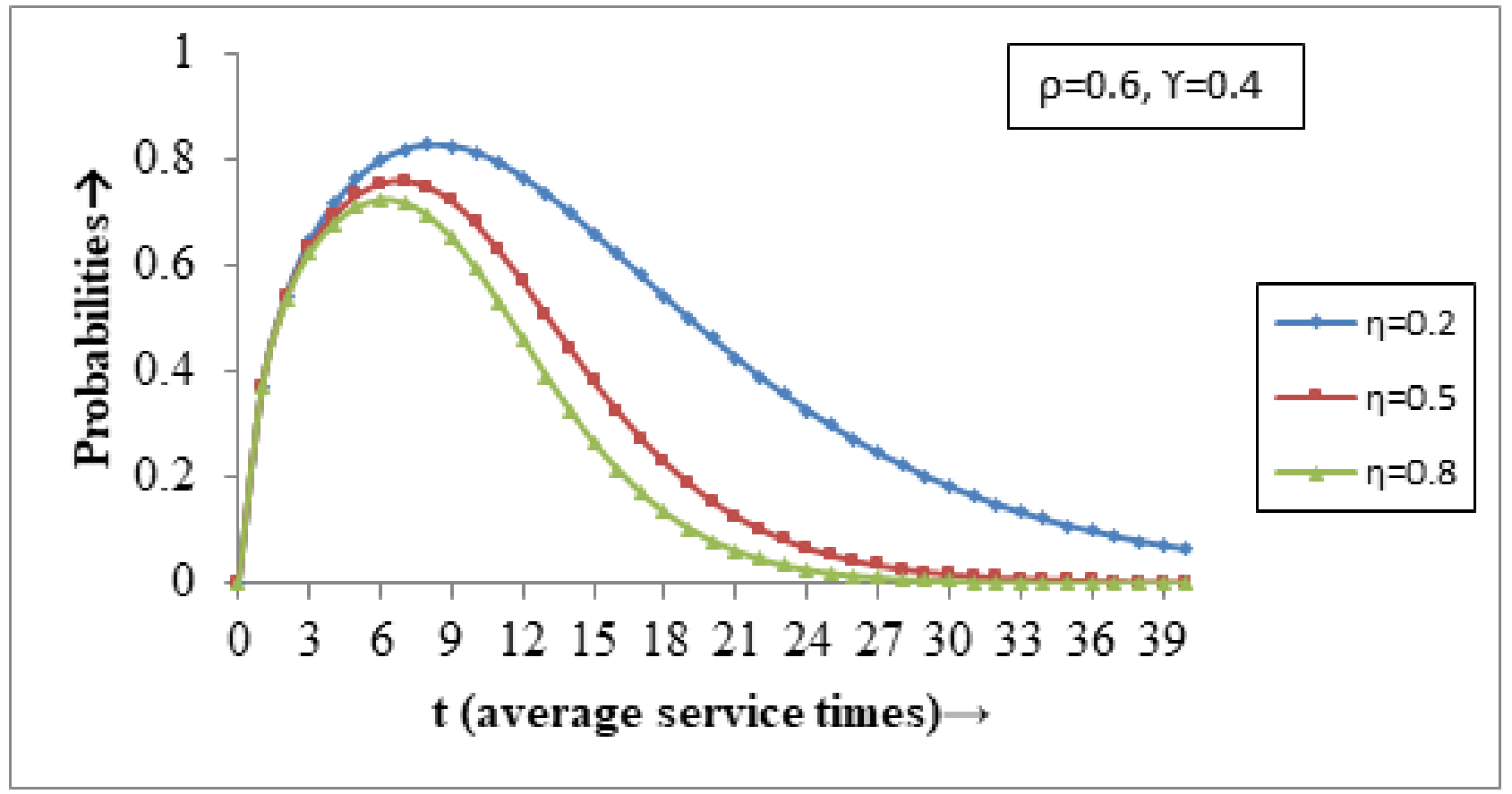

Fig 14. Effect of $\eta$ on System busy against $t$ (average service times)

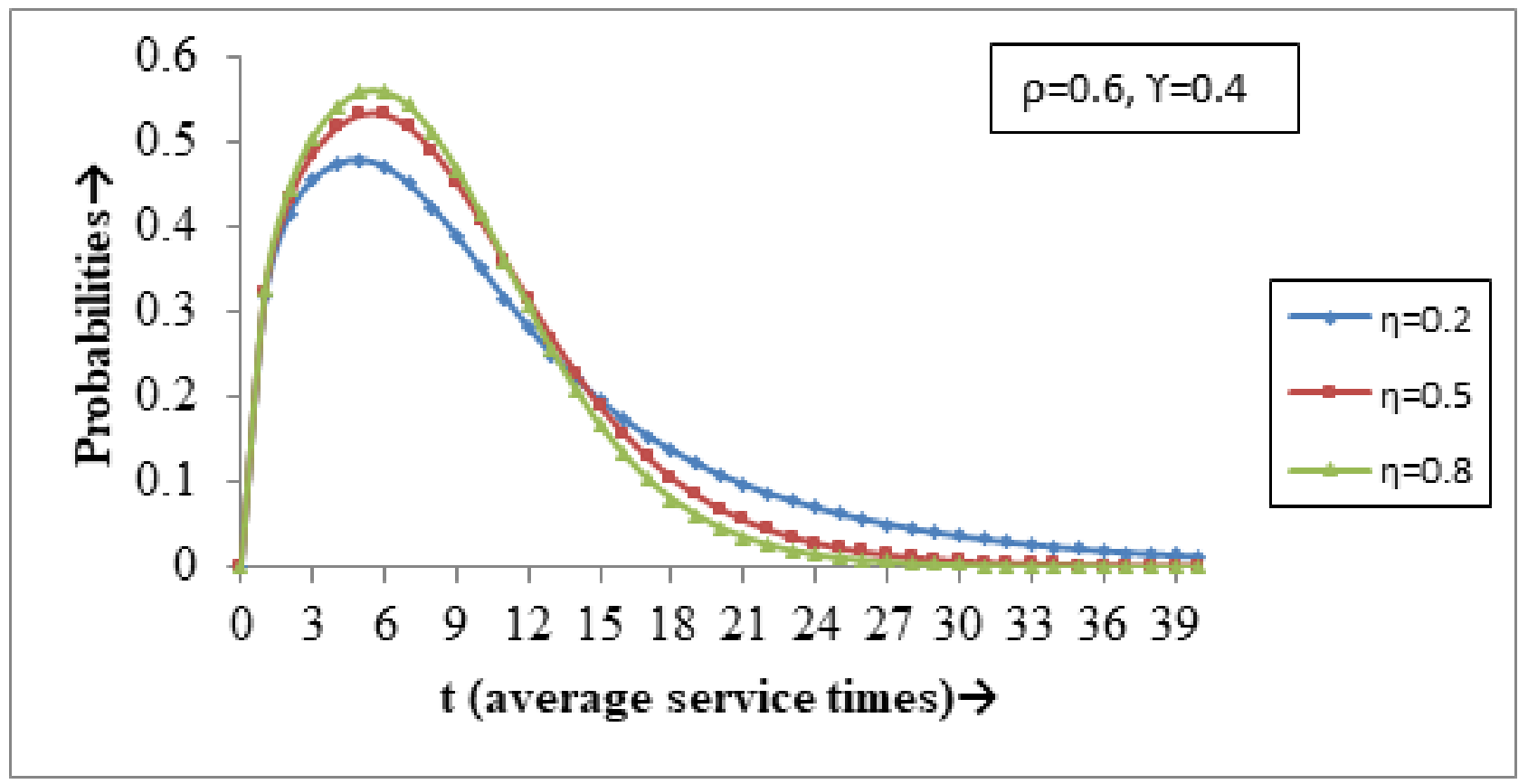

Fig 15. Effect of $\eta$ on Server busy against $\mathrm{t}$ (average service times) 
Figures 14 and 15, respectively show the probabilities of System busy and probabilities of Server busy plotted against time for various values of $\eta\left(\right.$ retrial intensity $\left.=\frac{\theta}{2 \mu}\right)$. For System busy the probabilities are higher for lower values of $\eta$. For Server busy the probabilities for initial to moderate values of $\mathrm{t}$ are lower for smaller $\eta$ but for higher values of $\mathrm{t}$ the trend changes, here the probabilities are greater for smaller $\eta$.

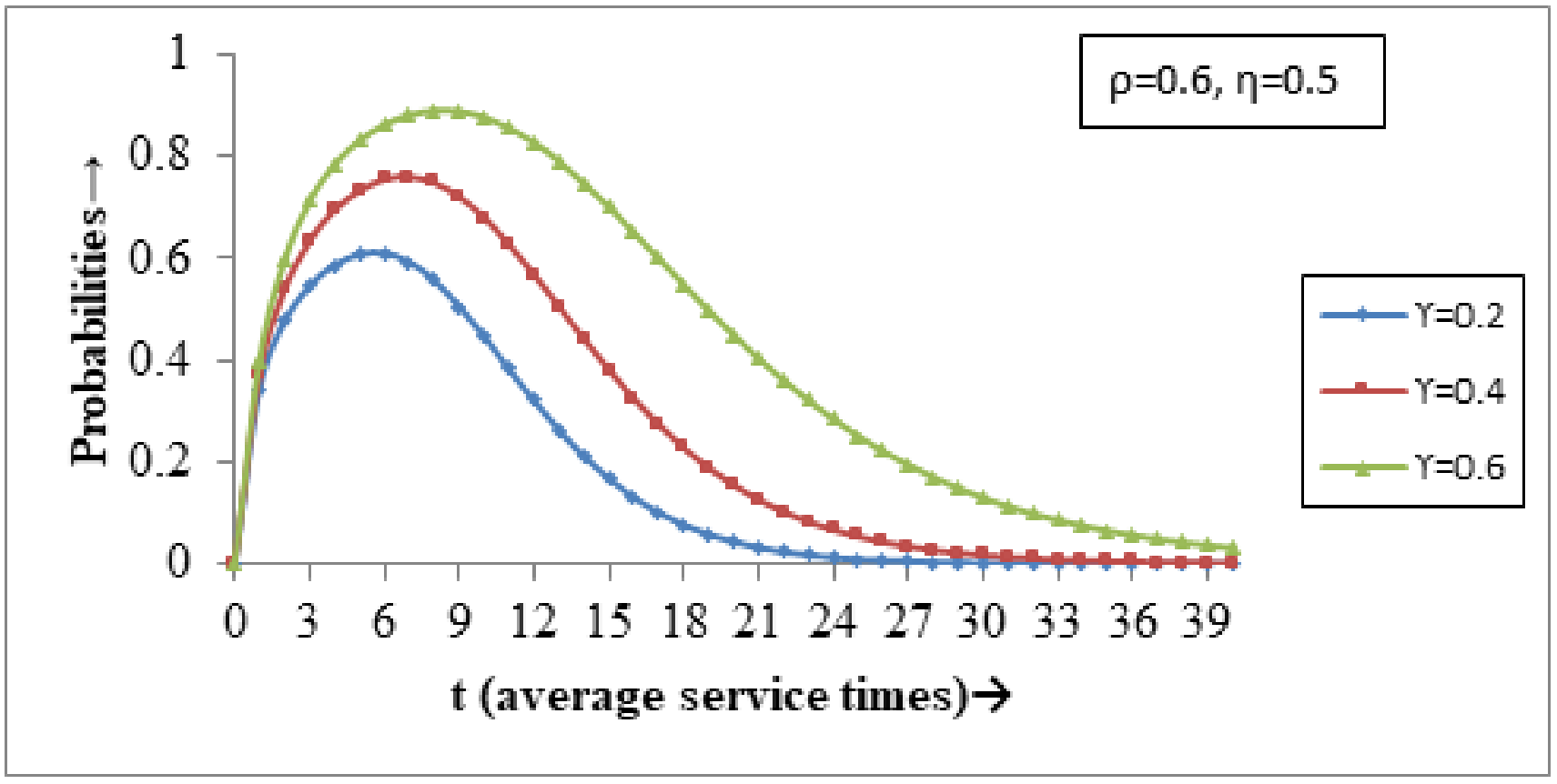

Fig 16. Effect of $\gamma$ on System busy against $\mathrm{t}$ (average service times)

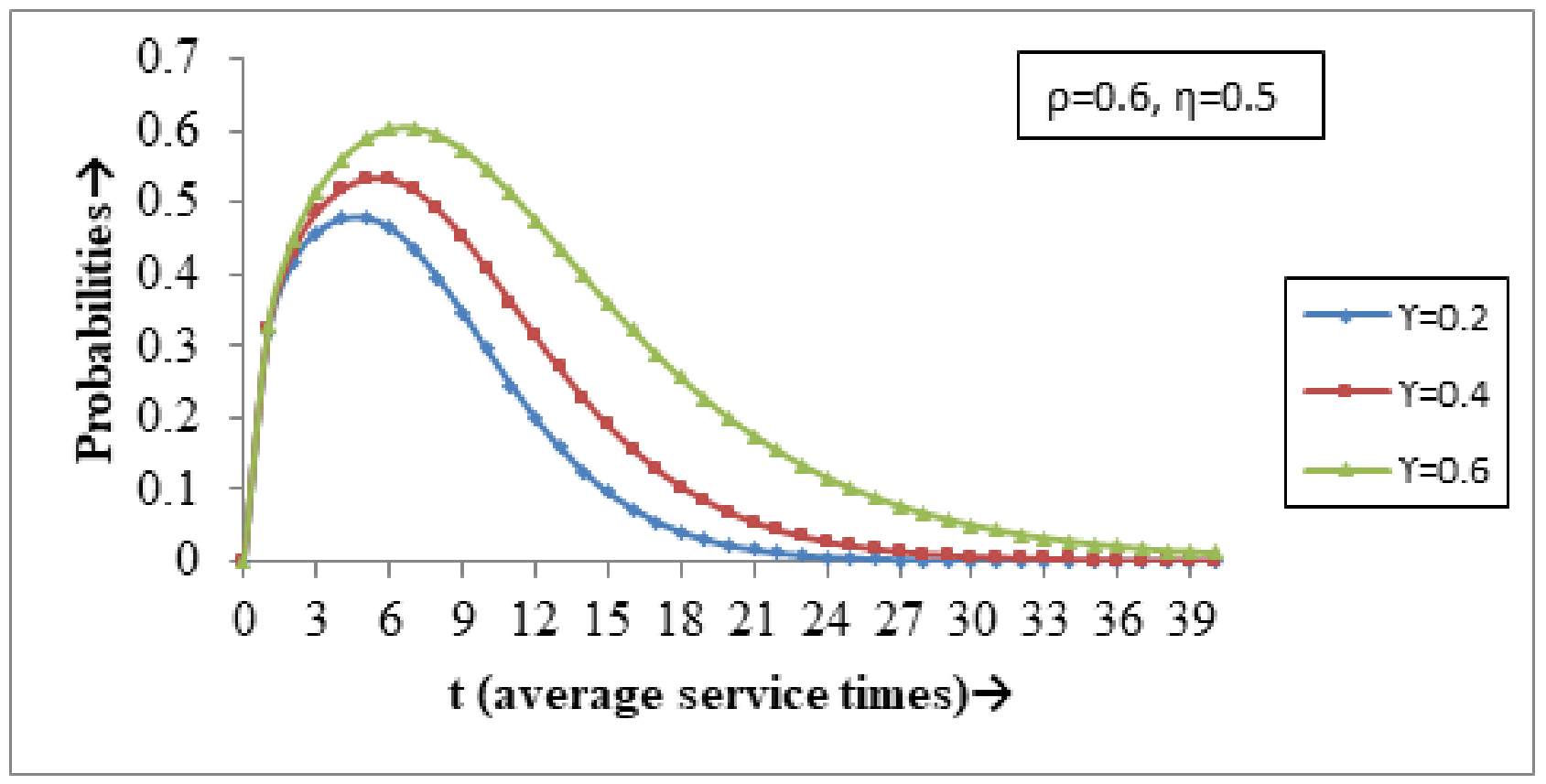

Fig 17. Effect of $\gamma$ on Server busy against $t$ (average service times) 
The probabilities of System busy and probabilities of Server busy are respectively plotted in Figures 16 and 17 for different values of $\gamma$ (probability of feedback). In both the graphs it is clear that the probabilities increase rapidly for the initial values of $t$ and then decrease gradually for higher $t$.

\section{Conclusion}

We studied two identical parallel servers with the concept of feedback. In previous research, queueing models with arrivals and departures from the orbit were considered but in present research we considered arrivals and departures from the system with the concept of feedback, in addition. We have obtained the time-dependent probabilities for exact number of arrivals and departures from the system when both servers are busy or free or one is busy. Several numerical results are obtained to check the effects of various parameters. This type of model is implemented in computer systems. The present model can serve as a base of future research for modeling practical situations where heterogeneous servers would be required.

\section{References}

1) Cohen JW. Basic problems of telephone traffic theory and the influence of repeated calls. Philips Telecommunication Review . 1957;18(2):49-100.

2) Yang T, Templeton JGC. A survey on retrial queues. Queueing Systems. 1987;2:201-233. Available from: https://dx.doi.org/10.1007/bf01158899.

3) Artalejo JR, Gomez-Corral A. A Computational Approach. In: and others, editor. Retrial queueing systems . 1999 ;p. XIII-318. doi:https://doi.org/10.1007/978-3-540-78725-9.

4) Artalejo J, Falin G. Standard and retrial queueing systems: a comparative analysis. Revista Matemática Complutense. 2002;15(1):101-129. Available from: https://dx.doi.org/10.5209/rev_rema.2002.v15.n1.16950.

5) Finch PD. Cyclic Queues with Feedback. Journal of the Royal Statistical Society: Series B (Methodological). 1959;21(1):153-157. Available from: https://dx.doi.org/10.1111/j.2517-6161.1959.tb00323.x.

6) Takacs L. 1963. Available from: https://doi.org/10.1002/j.1538-7305.1963.tb00510.x.

7) Lee YW. The M/G/1 feedback retrial queue with two types of customers. Bulletin of the Korean Mathematical Society. 2005;42(4):875-887.

8) Mokaddis G, Metwally S, Zaki B. A feedback retrial queuing system with starting failures and single vacation. Tamkang Journal of Science and Engineering. 2007;10(3):183-192.

9) Kumar BK, Vijayalakshmi G, Krishnamoorthy A, Basha SS. A single server feedback retrial queue with collisions. Computers \& Operations Research. 2010;37(7):1247-1255. Available from: https://dx.doi.org/10.1016/j.cor.2009.04.019.

10) Shekhar C, Jain M. Finite Queueing Model with Multitask Servers and Blocking. American Journal of Operational Research. 2013;3(2A):17-25.

11) Yang DY, Ke JC, Wu CH. The multi-server retrial system with Bernoulli feedback and starting failures. International Journal of Computer Mathematics. 2015;92(5):954-969.

12) Nobel R. Retrial queueing models in discrete time: a short survey of some late arrival models. Annals of Operations Research. 2016;247(1):37-63. Available from: https://dx.doi.org/10.1007/s10479-015-1904-7.

13) Pegden CD, Rosenshine M. Some New Results for theM/M/1 Queue. Management Science. 1982;28(7):821-828. Available from: https://dx.doi.org/10. $1287 / \mathrm{mnsc}$.28.7.821.

14) Indra R. Transient analysis of markovian queueing model with bernoulli schedule and multiple working vacations. Int J Comput Appl. 2011;20:43-48.

15) Singla N, Kalra S. Performance Analysis of a Two-State Queueing Model with Retrials Journal of Rajasthan . Academy of Physical Sciences. 2018;17(1 \& 2):81-100.

16) Singla N, Kalra S. A Two-State Multiserver Queueing System with Retrials. International Journal of Open Problems in Computer Science \& Mathematics. 2019;12(3):62-75.

17) Falin G, Templeton JG. Retrial queues. vol. 75. CRC Press. 1997.

18) Sztrik J. Basic Queueing Theory. 1986. 